\title{
In Situ Polymerization of a Conductive Polymer in Acellular Muscle Tissue Constructs
}

\author{
ANTONIO PERAMO, Ph.D., ${ }^{1}$ MELANIE G. URBANCHEK, Ph.D., \\ SARAH A. SPANNINGA, M.S., ${ }^{3}$ LAURA K. POVLICH, B.S.E., ${ }^{3}$ PAUL CEDERNA, M.D., ${ }^{2}$ \\ and DAVID C. MARTIN, Ph.D. ${ }^{1,3,4}$
}

\begin{abstract}
We present a method to chemically deposit a conductive polymer, poly $(3,4-e t h y l e n e d i o x y t h i o p h e n e)$ (PEDOT), on acellularized muscle tissue constructs. Morphology and structure of the deposition was characterized using optical and scanning electron microscopies (SEM). The micrographs showed elongated, smooth, tubular PEDOT structures completely penetrating and surrounding the tissue fibers. The chemical polymerization was performed using iron chloride, a mild oxidizer. Remaining iron and chlorine in the tissue constructs were reduced to acceptable metabolic levels, while preserving the structural integrity of the tissue. We expect that these acellular, polymerized tissue implants will remain essentially unmodified in cellular environments in vitro and in vivo because of the chemical and thermal stability of the PEDOT polymer depositions. Our results indicate that in situ polymerization occurs throughout the tissue, converting it into an extensive acellular, non-antigenic substrate of interest for in vivo experiments related to nerve repair and bioartificial prosthesis. We expect these conducting polymer scaffolds to be useful for direct integration with electronically and ionically active tissues.
\end{abstract}

\section{INTRODUCTION}

$\mathbf{T}$ HE INABILITY OF biological tissues to reestablish electrical connectivity after injury, illness, or tumor extirpation can significantly affect the recovery of function in affected individuals. This is a particularly difficult problem in peripheral nerves, where loss of electrical continuity leads to substantial motor and sensory deficits. Ultimately, these peripheral nerve deficits can reduce or eliminate an individual's ability to be gainfully employed or even their ability to perform typical activities of daily living. Unfortunately, not all of these peripheral nerve injuries can be repaired using biological tissues, and in situations involving amputations, there is no readily available and reliable way to provide an interface between the divided peripheral nerve stump and a prosthetic device. In these circumstances, an enhanced interface between the biologic tissue and an alloplastic material would provide substantial benefits through improved functional recovery after devastating injuries. To improve the integration of alloplastic materials with conductive tissues, we are exploring the possibility of using conducting polymers to provide an interface with reduced impedance for peripheral nerve regeneration.

Given the dielectric properties of most biological samples, a potential way to increase electrical conductivity is by performing modifications with conjugated polymers to produce a final product that can decrease impedance and is biocompatible, electrically and thermally stable, and non-toxic. Conjugated conducting polymers are of interest for tissue engineering, given that new biomaterials are required to support simultaneous tissue growth and electrical conductivity. ${ }^{1}$ Current innovations in the biomedical field include

\footnotetext{
${ }^{1}$ Department of Materials Science and Engineering, ${ }^{2}$ Department of Surgery, ${ }^{3}$ Macromolecular Science and Engineering Center, and ${ }^{4}$ Department of Biomedical Engineering, University of Michigan, Ann Arbor, Michigan.
} 
the use of polypyrrole ${ }^{2}$ and poly(3,4-ethylenedioxythiophene) $(\text { PEDOT })^{3}$ or its derivatives ${ }^{4}$ for neural prosthetics. PEDOT ${ }^{5,6}$ belongs to a group of conjugated polymers known for its good electrical conductivity due to electronic delocalization along the polymer backbone. This conductive polymer is environmentally and electrically stable. ${ }^{7,8}$ PEDOT has been shown to significantly reduce the impedance of biomedical electrodes designed for long-term implantation in living tissue. Its long-term chemical stability also makes it a suitable candidate as a conductive substrate for biological tissue repair. We approached this concept by modifying an acellular muscle construct. These modified acellular constructs could then be used for in vivo applications in peripheral nerve repair, as well as in applications in heart muscle, skeletal muscle, smooth muscle, and the central nervous system, where electrical conductivity is critical to function.

To this end, we have engineered PEDOT-modified acellular constructs, thinking that the enhanced electrical conductivity would be biologically advantageous to nerve prosthetic interfaces, nerve grafts, heart muscle patches, or denervated skeletal muscle reinnervation. In the biomedical area, attempts to use conductive polymers with lowlevel conductivity to help in peripheral nerve repair ${ }^{9}$ or to form hybrid materials in cardiac tissue ${ }^{10}$ have been reported, with successful neural recordings. For tissue repair, synthetic acellularized tissue substitutes have typically been engineered for skin, ${ }^{11}$ nerve, ${ }^{12}$ vocal fold, ${ }^{13}$ and other tissues, ${ }^{14}$ in part because the use of autografts in many surgical reconstructions is limited.

Possible solutions to the limited availability of autografts include the use of chemically acellularized, non-synthetic tissue allografts. Following the work of Rovak et al., ${ }^{15}$ after acellularization, peripheral nerves are left with a handful of molecules, including laminin, heparan sulfate proteoglycans, fibronectin, and collagen IV, present on the extracellular matrix (ECM) of the acellularized nerve construct. Immunologically hyporesponsive acellular biologic constructs have been engineered out of peripheral nerve and muscle. ${ }^{16}$ Acellularized muscle constructs are composed of cytoskeleton proteins, collagen, and proteoglycans that may be derived from allogenic tissues. Elimination of cellular elements from the tissue permits use of the ECM meshwork as an allograft for implantation without the need for immunosuppression.

In this report, we demonstrate the chemical in situ (within the tissue) polymerization of the conductive polymer PEDOT on acellular muscle tissue (AMT) constructs and characterize the acellular muscle tissue with polymerized PEDOT (AMT + PEDOT) constructs using microscopy, chemical analysis, and surface analysis methods. The purpose of our work was to investigate the optimum conditions for the production and manufacture of biologically inactive tissue with a conductive coating. We used chemical oxidation of the monomer 3,4-ethylenedioxylthiophene (EDOT) with a mild oxidizer $\left(\mathrm{Fe}^{3+}\right)$ in a reaction that takes place directly on the tissue. We also dedicated part of our efforts to reducing the presence of iron remaining in the tissue to levels compatible with in vivo iron metabolism. We used elemental and qualitative chemical analysis and X-ray photoelectron spectroscopy (XPS) characterization for this purpose.

\section{MATERIALS AND METHODS}

\section{Materials}

Iron chloride (III) was from Sigma-Aldrich (St. Louis, MO); EDOT (>97\%) monomer was from H.C. Stark (Newton, MA); potassium ferrocyanide, hydroxylamine hydrochloride, and 1-10-phenanthroline were from Acros Organics (Geel, Belgium); common reagents were from Fisher Scientific (Pittsburgh, PA).

\section{Tissue preparation}

AMT constructs were prepared from full-thickness mouse abdominal wall muscles, as previously described, ${ }^{17}$ and stored in a sodium azide solution. The acellularization method is presented in Table 1. Untreated AMT constructs were used as one type of control; in some cases, secondary

Table 1. Summary of Acellularization Methods including the Compositions and Timing

\begin{tabular}{lll}
\hline & \multicolumn{1}{c}{ Acellularizing reagent } & \multicolumn{1}{c}{ Time in reagent } \\
\hline A & glycerol $80 \% / 0.18 \% \mathrm{NaCl} / 0.05 \% \mathrm{NaN}_{3} / 0.1 \%$ & 2 days, *, refill 1 more day \\
& EDTA & $(3$ days total $)$ \\
$\mathrm{B}$ & sodium deoxycholate $4.2 \% / 0.05 \% \mathrm{NaN}_{3}$ & $* 1$ day \\
$\mathrm{A}$ & glycerol $80 \% / 0.9 \% \mathrm{NaCl} / 0.05 \% \mathrm{NaN}_{3} / 0.1 \%$ & $* 2$ days, $*$, refill 1 more day \\
$\mathrm{EDTA}$ & $1 \% \mathrm{SDS} / 0.05 \% \mathrm{NaN}_{3}$ & $* 2$ days \\
$\mathrm{D}$ & $3 \% \mathrm{Triton} \mathrm{X}-100 / 0.05 \% \mathrm{NaN}_{3}$ & $* 2$ days \\
$\mathrm{C}$ & $1 \% \mathrm{SDS} / 0.05 \% \mathrm{NaN}_{3}$ & $* 2$ days \\
$\mathrm{E}$ & $0.05 \% \mathrm{NaN}_{3}$ & $*$ Store in this solution indefinitely \\
\hline
\end{tabular}

*Between each step, solutions were drained, tissues were blotted and rinsed with water. $\mathrm{NaCl}$, sodium chloride; EDTA, ethylenediamine tetraacetic acid; SDS, sodium dodecylsulfate, $\mathrm{NaN}_{3}$, sodium azide. 
controls were prepared with AMT constructs containing iron chloride $\left(\mathrm{FeCl}_{3}\right)\left(\mathrm{AMT}+\mathrm{FeCl}_{3}\right)$ but no EDOT; test samples were AMT constructs chemically polymerized with PEDOT $($ AMT + PEDOT), as described later.

\section{Chemical oxidation of EDOT}

In situ polymerization of EDOT was performed using a method described elsewhere. ${ }^{18}$ First, to eliminate the sodium azide from the untreated AMT constructs, samples were abundantly washed and sonicated in deionized water with repeated rinses and change of sonicated solutions with fresh solvent. Then, as shown in Figure 1, EDOT was in situ polymerized on mouse AMT constructs using ferric chloride oxidant in the presence of ethanol. The AMT construct was conditioned at $-20^{\circ} \mathrm{C}$ for $20 \mathrm{~min}$ in ethanol and EDOT in a concentration to keep the molar ratio EDOT: $\mathrm{FeCl}_{3}$ as 1:4. Then $\mathrm{FeCl}_{3}$ was added and thoroughly mixed and kept at $-20^{\circ} \mathrm{C}$ for $40 \mathrm{~min}$ to slow the polymerization. The AMT constructs were air dried overnight in a fume hood, allowing the monomer to polymerize. Some AMT constructs were subjected to 1 cycle of polymerization (AMT + PEDOT1), the others were subjected to 6 cycles of polymerization (AMT + PEDOT6).

\section{Sonication}

Sonication was performed to help eliminate excess iron and chlorine from the AMT constructs after polymerization, and different times were tested to achieve this goal.
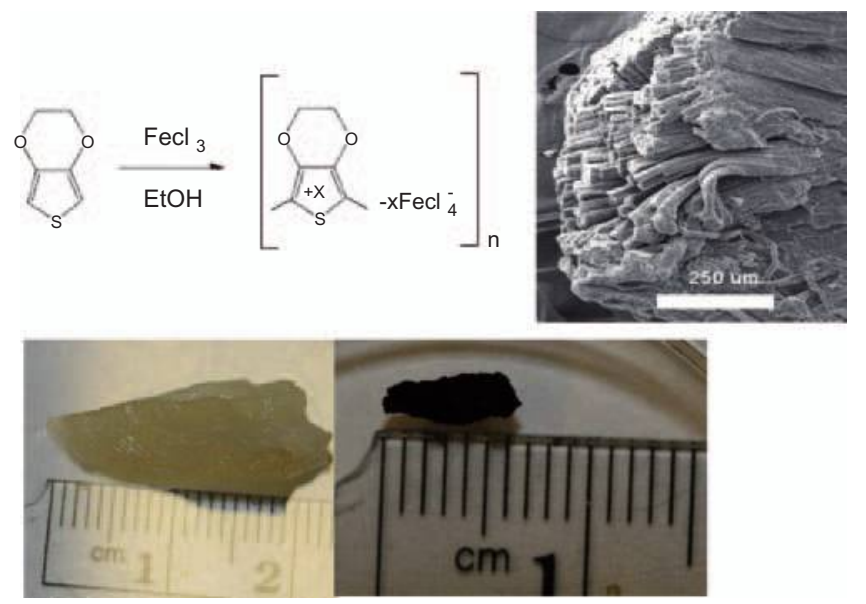

FIG. 1. Top left, chemical oxidation of 3,4-ethylenedioxythiophene. Top right, scanning electron microscope image showing the fibrous morphology of the mouse acellular muscle tissue (AMT) construct (not polymerized). Bottom, optical image of wet AMT construct before polymerization (left) and dry polymerized AMT + poly(3,4-ethylenedioxythiophene construct subjected to 6 cycles of polymerization (right). These images belong to different samples and are not representative of shrinking ratios. Color images available online at www.liebertpub.com/ten.
Each polymerization cycle was performed as described above. Between cycles, the AMT constructs were sonicated for $2 \mathrm{~min}$ in ethanol. After the final polymerization cycle, the AMT constructs were rinsed with ethanol, sonicated for $30 \mathrm{~min}$ in ethanol with solvent changes every 5 minutes, rinsed with water, and stored in air before use in subsequent experiments.

\section{Construct characterization}

Optical microscopy. Optical microscopy observations were performed using a Nikon Optiphot POL (Nikon, Tokyo, Japan) with an attached Spot RT (Diagnostic Instruments, Sterling Heights, MI) digital camera for image acquisition. Thin $(2-3 \mu \mathrm{m})$ frozen sections of the sonicated AMT, AMT + PEDOT1, and AMT + PEDOT6 constructs were obtained.

Scanning electron microscopy. Observation of morphology and microstructure of tissue coating in different conditions (sonicated AMT, AMT + PEDOT1, and AMT + PEDOT6 constructs and non-sonicated AMT + PEDOT6) was performed with a Philips/FEI XL30 ESEM instrument (FEI Company, Hillsboro, OR). SEM micrographs were taken with accelerating voltage of $15 \mathrm{kV}$ and spot size of 3 . For SEM observation, a thin film of gold was deposited using a common gold sputterer.

Analytical characterization. Positive presence of $\mathrm{Fe}^{3+}$ was initially determined using potassium ferrocyanide (Prussian Blue). Alternatively, the presence of $\mathrm{Fe}^{2+}$ was detected with 1-10-phenanthroline ${ }^{19}$ independently and after use of hydroxylamine hydrochlorate. These were done to provide an initial estimate of the time frame required for sonication. Because Prussian Blue formation is not totally selective for iron, an additional control was performed by oxidizing available $\mathrm{Fe}^{3+}$ present in the sample with hydroxylamine hydrochloride followed by the use of 1-10phenanthroline to identify iron in the form of a red chelate, $\left[\mathrm{Fe}(\text { phen })_{3}\right]^{2+}$. For elemental analysis, duplicate samples were examined for total amount of iron using a PerkinElmer Optima 2000 ICP-OES (Inductively Coupled PlasmaOptical Emission Spectrometer) instrument (PerkinElmer, Waltham, MA), which is routinely used to determine iron in biological samples. For iron, the results are given in weight percentage and represent, for each construct type, an average of 3 different readings at 2 different spectral wavelengths: $238.204 \mathrm{~nm}$ and $239.562 \mathrm{~nm}$.

XPS characterization. Duplicate samples were also analyzed using XPS. XPS measurements were performed using a Kratos Axis Ultra DLD XPS (Kratos Analytical Ltd., Manchester, UK). A monochromatic aluminum X-ray source was used. All spectra are referenced to the $\mathrm{C}-\mathrm{C} / \mathrm{C}-\mathrm{H}$ binding energy at $285 \mathrm{eV}$. 


\section{RESULTS}

\section{Construct preparation and chemical polymerization}

We prepared the AMT constructs by polymerizing with $\mathrm{FeCl}_{3}$ as an oxidant, leaving the polymer in the p-doped (conductive) state. This method resulted in the deposition of a black compound, PEDOT, on the tissue, as observed in Figure 1. Our AMT constructs (acellular mouse abdominal muscles) were composed of bundles of fibers (Fig. 1, top right) and had a white yellowish coloration before polymerization and a dark black appearance after polymerization (Fig. 1, bottom). The image in Figure 1 representing the polymerized AMT construct (Fig.1, bottom right) was taken when the tissue was completely dry (no sections were taken in this condition), whereas the images in Figure 2 show tissue humidified after cutting frozen sections; thus it is not possible to directly compare the fiber size. After the chemical deposition of PEDOT, the previously pliable AMT construct became stiff and brittle. The shrinking of the tissue is readily observable by the naked eye, suggesting that the polymer may have permeated the whole AMT construct rather than just forming a thin layer surrounding the fibers. This is mostly due to the drying process, but we believe that there is a contribution to this process due to polymerization that we have not yet quantified precisely. There is also the potential influence of volume changes of the polymer itself due to the exchange of counterions during oxidation. ${ }^{20}$

\section{Optical characterization}

The permeation of PEDOT into the AMT construct is observed in the optical images shown in Figure 2. The images were taken from $2-\mu \mathrm{m}$ longitudinal and 3- $\mu \mathrm{m}$ transverse sections of AMT, AMT + PEDOT1, and AMT +
PEDOT6 constructs. The anisotropic structure of the AMT construct, albeit with a directional preference along the fibers, results in the anisotropic structures of the polymerization. What appear to be structural inhomogeneities of the AMT construct are visible as a result of the color provided by the polymer and are more visible when the amount of polymer is smaller, for instance in the images of AMT + PEDOT1 constructs with 1 cycle of polymerization. These optical images indicate that further PEDOT deposition, as indicated by the darker color in Figure $2 \mathrm{E}$ and F, occurred during multiple polymerization cycles.

\section{Scanning electron microscopy}

Observations of morphology of the AMT constructs before and after PEDOT deposition were performed using SEM. Figure 3 shows aspects of the morphology of the polymerization on the AMT construct, demonstrating that PEDOT coatings grew thicker with increasing polymerization cycles. All samples shown in these micrographs were sonicated as described in Materials and Methods, with the exception of some AMT + PEDOT6 constructs (micrographs C and G), which were not sonicated. The top 2 micrographs (A and E) correspond to control AMT constructs at low and higher magnification. Micrographs $\mathrm{B}$ and $\mathrm{F}$ correspond to sonicated AMT + PEDOT1 constructs. These 2 micrographs demonstrate that the initial morphology of chemically polymerized PEDOT is composed of globular particles resembling the spherical particles obtained in electrochemical polymerizations. ${ }^{4}$ However, a close observation of Figure $3 \mathrm{~F}$ shows that some areas remain sparsely polymerized while others are densely polymerized, indicating that, to obtain good substrate modification and surface coverage, it was necessary to perform additional cycles of polymerization.

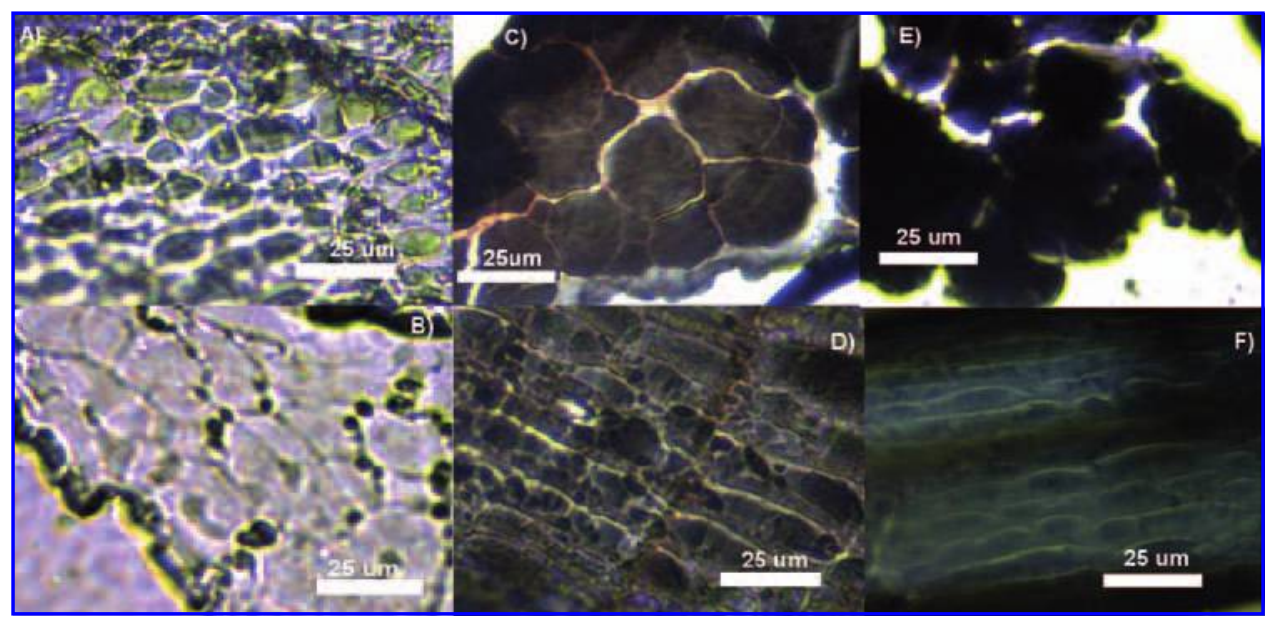

FIG. 2. Optical micrographs of mouse abdominal acellular muscle tissue (AMT) samples. Top row are images taken from 3- $\mu \mathrm{m}$ transverse sections; bottom row are images taken from 2- $\mu$ m longitudinal sections, all at $40 \times$ magnification. In both rows, from left to right: (A) and (B) sonicated control AMT constructs; (C) and (D) sonicated AMT+ poly(3,4-ethylenedioxythiophene construct subjected to 1 cycle of polymerization (PEDOT1) constructs; and (E) and (F) sonicated AMT+PEDOT6 constructs. Color images available online at www.liebertpub.com/ten. 

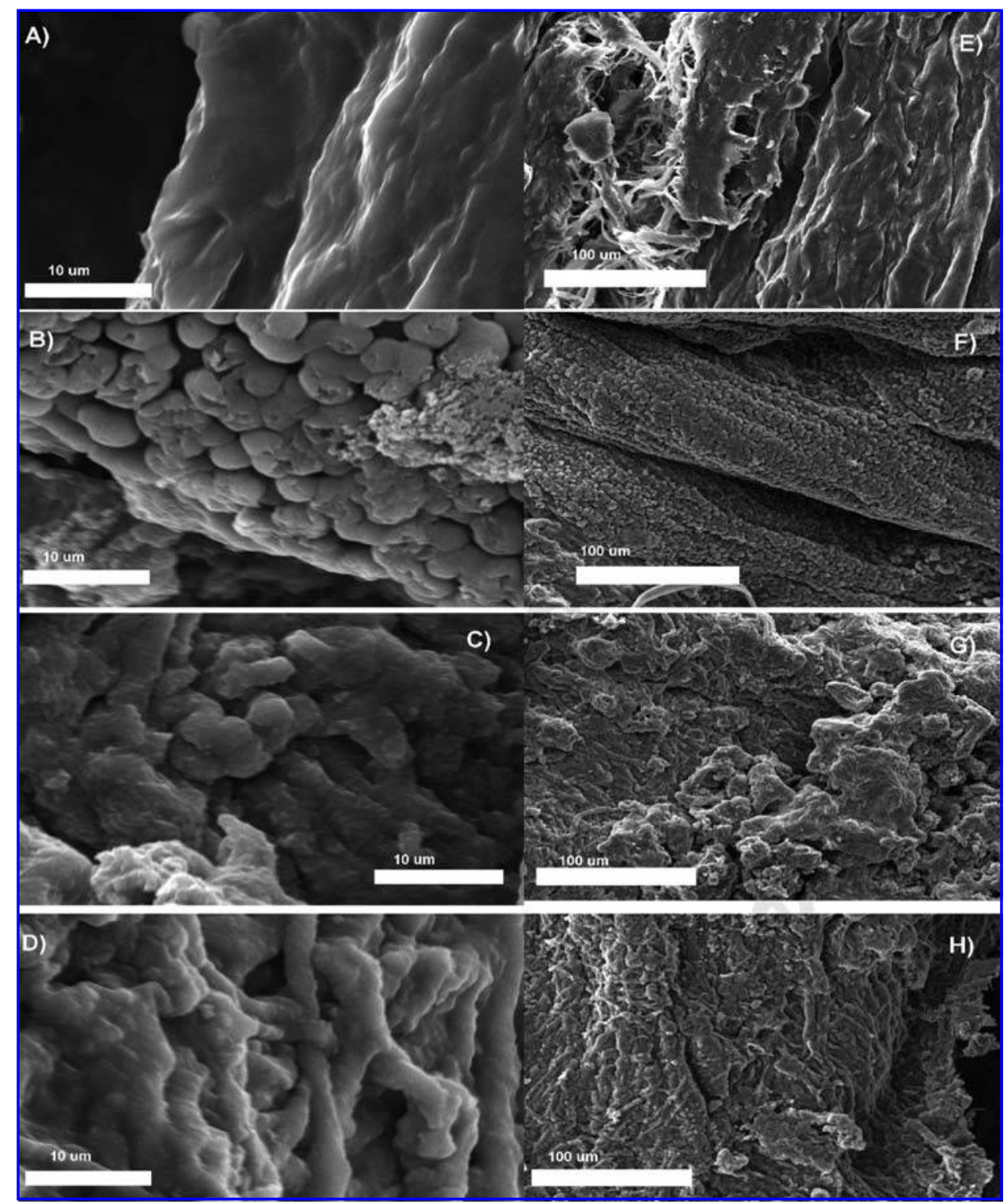

FIG. 3. Scanning electron microscopy micrographs of mouse abdominal wall acellular muscle (AMT) constructs. Left column micrographs are 2000× magnification; right column are 250×. Micrographs (A) and (E) show a control AMT construct, (B) and (F) an AMT + poly(3,4-ethylenedioxythiophene construct subjected to 1 cycle of polymerization (PEDOT1) construct, (C) and (G) a nonsonicated AMT + PEDOT6 construct, and (D) and $(\mathbf{H})$ a sonicated AMT + PEDOT6 construct. As observed, PEDOT in the AMT constructs coats the muscle filaments and fills the areas between them by infiltration, working as a filler of the construct.

Striking morphological changes can be observed at the sixth cycle of polymerization (Fig 3C, D, G, and H). The globular particles converted into tubes and long tree root-like fibular structures, as seen in Figure $3 \mathrm{H}$.

\section{Chemical analysis and XPS}

The results indicated that control AMT constructs did not have substantial amounts of iron present, suggesting that the major part of iron detected later came from the chemical oxidation procedure. All non-sonicated constructs gave positive identification of $\mathrm{Fe}^{2+}$ and $\mathrm{Fe}^{3+}$, whereas sonicated constructs showed presence of some iron, although the intensity of the color produced by the chromophore decreased with longer sonication times. To facilitate iron elimination, sonication was performed in ethanol for 2 min between polymerization cycles. Iron was difficult to observe with potassium ferrocyanide in samples that had been sonicated for $30 \mathrm{~min}$ in ethanol, with solvent changes at least every $5 \mathrm{~min}$, but was still detected using hydroxylamine hydrochloride and 1-10-phenanthroline. This indicated that most of the iron not added in the dopant backbone was reduced during polymerization. Thirty min was considered the minimum time necessary for sonication. 
The XPS technique was sensitive enough to provide accurate information on the presence of the iron on the surface of the AMT construct within the outermost 2- to 5-nm layer. XPS is only useful for analysis of the outermost layers of the material; for this reason, to test for the presence of oxidizer inside the AMT constructs, we additionally performed elemental analysis of whole constructs, which is discussed below. We did not test the amount of monomer remaining inside the construct because the chemical oxidation is performed with excess oxidant over monomer.

The samples included in the spectra (Fig. 4) are sonicated control AMT constructs (spectrum E), sonicated AMT + $\mathrm{FeCl}_{3}$ (spectrum C), sonicated AMT + PEDOT1 (spectrum B), sonicated AMT + PEDOT6 (spectrum D), and nonsonicated AMT + PEDOT6 (spectrum A). No iron peak was present in control AMT constructs (spectrum E), but there were strong iron peaks for non-sonicated AMT + PEDOT6 constructs (spectrum A) and for sonicated AMT + PEDOT1 constructs (spectrum B) $(\sim 712 \mathrm{eV})$. The spectrum of $\mathrm{AMT}+\mathrm{FeCl}_{3}$ showed a less-intense iron peak than nonsonicated AMT + PEDOT1 constructs. This effect was due to the doping process that traps the iron in the polymer. The peak intensities were lower for AMT + PEDOT1 constructs sonicated for $30 \mathrm{~min}$, practically at the levels of control AMT constructs. When present, the intensity of the iron peaks was greater than of the sulfur peaks from PEDOT, probably because, during oxidation, a 4-M excess of iron is used.

In the XPS spectra, the presence of the sodium peak in control AMT constructs but not on the other constructs

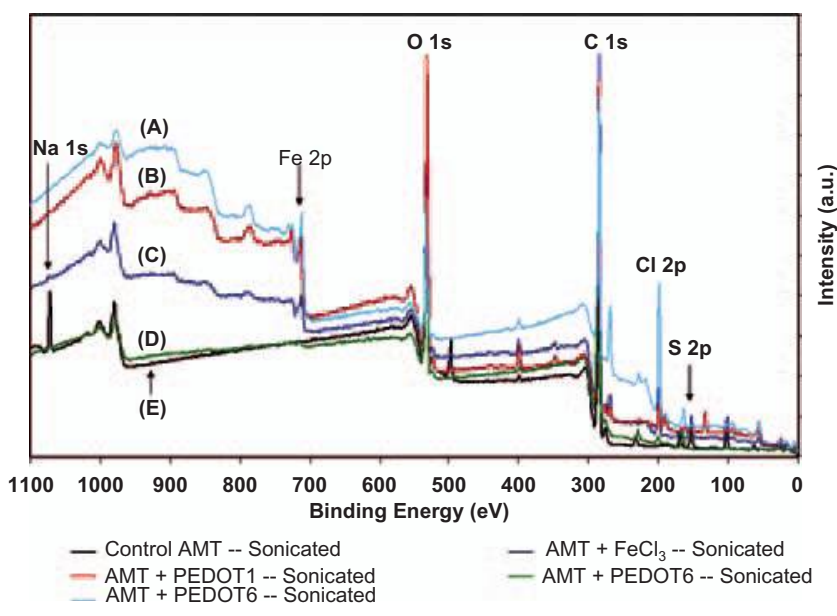

FIG. 4. X-ray photoelectron spectroscopy spectra of mouse acellular muscle tissue (AMT) samples: (A) non-sonicated AMT + poly(3,4-ethylenedioxythiophene construct subjected to 6 cycles of polymerization (PEDOT6) construct, (B) sonicated AMT + PEDOT1 construct, $(\mathbf{C})$ sonicated AMT + iron chloride construct, (D) sonicated AMT+PEDOT6 construct, and (E) control AMT construct. Color images available online at www.liebertpub .com/ten. indicated that some sodium was still present on the AMT construct after the initial $2 \mathrm{~min}$ of sonication. However, the peak disappeared with the polymerization treatment and further sonication procedures. For chlorine, there was a reduction in the peak intensity; the non-sonicated samples had a substantial presence of chlorine on the surface, but after sonication, the chloride peak almost disappeared.

Elemental analysis of control AMT constructs gave an average iron concentration, in percentage weight, of $0.43 \%$, AMT + PEDOT1 constructs showed a $2.97 \%$ iron content, and AMT + PEDOT6 constructs had a $3.32 \%$ iron content. Given the cumulative amount of iron used along the 6-time polymerization cycle, a 6-fold average increase with respect to the control samples seems a fairly reasonable increase after dedoping.

\section{DISCUSSION}

We have shown that the conductive polymer PEDOT can be chemically polymerized in a biological tissue sample. Polymerization of PEDOT on biomedical devices has typically been performed electrochemically, a process that cannot be achieved directly over biological tissue because of the lack of substrate conductivity. For bulk, massive structures or mass production, chemical oxidation is a more suitable method for performing the polymerization. ${ }^{21}$ For biological applications, chemical polymerization may be much better suited, in particular in cases in which the polymerized samples do not need to have low resistivities, as is typically the case when PEDOT is used in industrial applications.

Chemical polymerization of EDOT can be performed using several methods and oxidants. The classical method employs oxidizing agents such as $\mathrm{FeCl}_{3}{ }^{22}$-which is a hazardous material—or iron (III) $p$-toluene sulfonate, ${ }^{23}$ which is a common oxidant but has an important drawback. We did not use this oxidant because some of its derivatives, namely esters, are considered to be potential alkylating agents. Typically, PEDOT oxidation is performed in the presence of alcohols, and the presence of any alcohol during polymerization or later during implantation may induce the formation of sulfonic acid esters. In particular, methyl and ethyl methanesulfonate esters are known genotoxins and carcinogens in rats and mice ${ }^{24}$ whereas toxicity of $p$-toluene sulfonate esters has been generally established. ${ }^{25}$

Other oxidants have been used, including ferric sulfate ${ }^{26}$ or more recently enzymes. ${ }^{27}$ In our selection, we took into account the final use of the tissue, namely its possible implantation in animals and humans, and the simplicity of preparation. Therefore, we used an oxidant that produced reaction by-products that could be partially eliminated from the tissue after polymerization, resulting in the partial dedoping of the polymer. The solvent used was ethanol because it offers good conditions for solubilization of EDOT monomer and iron chloride, although butanol could have also been used. 
The formation of small particles after the first polymerization, as seen in Figure 3B and F, may be due to the tendency of initial formation of oligomers, instead of long polymerized chains. Additional polymerization cycles may help in elongating the polymer chains, as seen in Figure 3D and $\mathrm{H}$. Although these elongated fibers are also visible in the non-sonicated sample (Fig. 3C, G), they are hidden and buried underneath a layer of deposited material, probably composed of unreacted EDOT monomer, oligomers, and oxidant molecules. Sonication somewhat eliminates this layer, producing a partial dedoping of the polymerization. Apart from the elimination of iron and chlorine, this procedure has the additional advantage of smoothing the surface, reducing its roughness and asperity. The morphology of the constructs are seen to maintain the 3-dimensional form provided by the original piece of acellular muscle. Muscle fiber orientation is maintained during polymerization in all cases. In general, morphological changes are visible from the control to polymerized samples but macroscopically do not change with the number of polymerizations. Microscopically, there is aggregation of polymer and the conversion of globular PEDOT into elongated tubes. Qualitatively, we observed a reduction in volume of the constructs from wet non-polymerized to dry polymerized, but the original wet size was not recovered after submersion in water, presumably because the polymer prevented water from reaching deep into tissue. We plan to analyze the exact amount of polymer deposited in the constructs by using thermogravimetric techniques that will provide the basis for precise quantitative measurements of morphological changes.

The amount of iron on the surface appears to be lower after 6 polymerizations, as determined using XPS, than after 1 polymerization, even when the total iron concentrations, from elemental analysis data, were similar. This means that the majority of iron present in the AMT constructs is buried inside the tissue, trapped inside the PEDOT. When used in vivo, this may permit a slow release of iron from the construct into the surrounding ECM in the event that the dedoping process continues after implantation.

We introduce now a discussion on the stability of PEDOT and its usefulness as a possible protein-masking agent, in addition to its known use as a conductive substrate. Given the long-term purpose of this research, namely in vivo implantation of acellular AMT constructs modified with conductive polymers, the emphases were put on the chemical stability and composition of the polymerized AMT constructs and the elimination of iron and chlorine. We found that, when EDOT was chemically oxidized and deposited on the AMT construct, it formed an elongated polymeric meshwork that surrounded the fibers but that the polymerization also took place inside them, instead of just as a thin layer on the outer surface of the sample, as demonstrated in the optical and SEM images. We consider that the polymer fills the constructs, in addition to coating the filaments, as demonstrated in the optical images in Figure 2. From this point of view, the deposited polymer works as a filler of the tissue, not just as a coating. The reason for this effect may be related to the porosity of the material. Materials that are typically used as substrates for chemical or electrochemical oxidation of PEDOT, like platinum electrodes or fabrics, present crystalline or fiber mesh structures where EDOT monomers cannot penetrate or are unreachable for oxidation, thus keeping the deposition of the polymer outside their structures. In contrast, the small EDOT monomer and the oxidant, in this case a non-bulky $\mathrm{FeCl}_{3}$ group can permeate the loose structure of biological samples, particularly when submersed in solvents. There seems to be good preservation of the AMT construct structure after polymerization. In our case, this is not a primary concern, in contrast with other substrates like fabrics, in which the structural integrity of the mesh must be preserved. It is unclear how this internal polymerization may affect the conductivity of the AMT construct differently from typical thin layer polymerizations, because in this case it does not seem possible to polymerize exclusively on the outer surface of the acellular construct. It is also unclear how this internal deposition may improve the longevity of the polymerization. Although our deposition methods are not the same as those studied by WintherJenssen, ${ }^{28}$ there generally seems to be an intrinsic increase in the impedance of PEDOT thin films over time. Bulk, chemical polymerizations make the total amount of monomer oxidized during polymerization difficult to control. Variations in the iron chloride:monomer ratio could provide a first method to control the amount of dopant and hence, possibly, the impedance.

There is a possibility that these polymerized structures could eventually come off the AMT construct fibers (i.e., by solubilization or degradation) and that isolated EDOT monomers or whole PEDOT polymer sections could be released into the ECM of the areas surrounding the in vivo implants, although we believe that monomer release and PEDOT removal will not occur because of the chemical stability of PEDOT. The PEDOT polymer backbone is not of direct biological origin; thus its degradation by mammalian enzymes may be difficult or impossible. In essence, because PEDOT appears to be covering the AMT construct completely, it is masking the AMT construct proteins underneath. As a result, slow degradation, if any, of the polymer is expected, thus extending the working lifetime of the implant. In this sense, we propose here that PEDOT will work to protect the AMT construct proteins from enzymatic degradation after implantation. ${ }^{29}$ An additional advantage is that the possible remaining antigenicity of the acellular construct, if any, should be reduced.

Because the AMT construct itself has an inherently porous, fine structure that is determined by the geometry of the original tissue, we expect it to serve as a robust platform for tissue regeneration and not promote the formation of a foreign body or encapsulation response that is seen when large, insoluble objects are implanted in tissue. For use in peripheral nerve repair, we envision that the construct will 
more rapidly and successfully guide regenerating nerve axons of neurotomized nerve to the transected distal nerve stump or to muscle fibers, where they will terminate in neuromuscular junctions. These outcomes will result in acceptable nerve function rather than allowing the axons to grow and become painful neuromas. At this point, we do not envision any remodeling of the AMT constructs.

Next, we briefly discuss the difficulty of the chemical degradation of PEDOT. Chemically, EDOT is composed of an ethylenedioxy bridge group and a thiophene ring. After polymerization, an attack on the ethylenedioxy bridge group will essentially convert the PEDOT backbone into a modified polythiophene backbone. To a certain degree, that may affect the mechanical stability of the structure, leading to solubilization or degradation of the backbone. It may also reduce the electrical conductivity due to a reduction of the distribution of the total charge because, in the p-doped state, there is a stabilizing effect of the polymer produced by the electron donor dialkoxy groups. ${ }^{30,31}$ However, substituents in the 3 and 4 positions of the thiophene ring prevent $\alpha$ and $\beta$ coupling during polymerization, lowering the oxidation potential of the monomers and stabilizing the oxidized form of the polymers, which are not as sensitive to nucleophilic attack as the unsubstituted materials. ${ }^{32}$

Unsubstituted thiophene belongs to a group of conjugated compounds that are known to be resistant to ring opening, subsequent desulfurization, and degradation. ${ }^{33}$ Chemical modification of isolated thiophene rings is common but not after formation of the polymer backbone. From a biological perspective, opening of the thiophene ring may produce undesirable degradation compounds. For instance, sulfur oxidation in thiophene can happen when the molecule or its derivatives are in monomeric form in biological environments. ${ }^{34}$ In the case of doped PEDOT, however, the geometry of the monomer changes and is stabilized, first, after formation of the ethylenedioxy bridge group and, second, after oxidation and formation of the polymer backbone, making the polymer intractable and difficult to attack due to steric hindrances.

So far, we have discussed the possible outcomes of the chemical degradation of PEDOT in the AMT constructs. Although, as we have mentioned, our initial work has the intention of describing a method for their production, showing that the manufacturing process maintains the intact structure of the tissue and establishing a procedure for elimination of the remaining oxidant, we are currently designing experiments to test the cytotoxicity and conducting properties of the construct in vitro. We are conducting our preliminary work with a mixed culture of myoblasts and fibroblasts. Preliminary results indicate that these cells grow and replicate on the plates immediately adjacent to PEDOTmodified materials, providing early indication that the AMT constructs have no or low cytotoxicity.

After polymerization and sonication, the amount of $\mathrm{FeCl}_{3}$ remaining in the AMT construct, trapped as dopant material deep inside or on the surface, was determined. The process of iron and chlorine elimination constitutes an attempt to de-dope the polymer, and it has been proven in the past that it is not possible to eliminate the dopant completely from chemically oxidized PEDOT polymers. ${ }^{35}$ As our results indicate, iron was mainly confined inside the AMT construct, and the amount remaining was low, but in any case, we decided that it was necessary to establish a method to minimize the presence of iron in the samples implanted in animals or humans that could be released and accumulate in certain organs, particularly the liver, spleen, and bone marrow. This minimization was more necessary because of the peculiarity of the metabolism of iron in humans, which occurs in a virtually closed system. ${ }^{36}$ Typically, little iron is absorbed from the diet $(\sim 1-2 \mathrm{mg})$, and the excretion in the urine is minimal, the absorption being strictly controlled by the intestine to prevent toxic accumulation in organs. After implantation of an acellular construct, iron in the sample may be released and diffused as if it were given parenterally or intravenously, depending on the location of the implanted AMT construct and the proximity to microvasculature, thus incorporating quickly intro the bloodstream and accumulating, in final state, in parenchymal cells of liver and endocrine tissues.

Although iron can be present in the samples as unreacted $\mathrm{Fe}^{3+}$, our results indicate that almost all iron is present as dopant or is reduced during the polymerization process, and thus most of the initial iron in the AMT construct will be in its reduced state. Following the results of the elemental analysis, and assuming that the total weight of a typical implanted acellular AMT construct is on the range of 5 to $15 \mathrm{mg}$, the absolute amount of iron implanted lies in the range of 0.16 to $0.50 \mathrm{mg}$. Thus, at a maximum, the implanted AMT construct will contain an amount of iron equal to half of one day's absorption from the diet in humans, which is a safe amount, especially when compared, for example, with iron overload in transfusions, given that each unit of blood contains 200 to $250 \mathrm{mg}$ of iron. Overall, the results of the analysis of iron remaining on the AMT construct samples give a strong indication that they will be safe for implantation.

In summary, in this report, we present a method for the chemical polymerization of the conductive polymer PEDOT in acellularized AMT constructs. We determined that a good method of preparing the samples for their possible use as acellular constructs for implantation in vivo was to polymerize with iron chloride as the oxidant. The polymer was partially de-doped using sonication for $30 \mathrm{~min}$ in ethanol, reducing iron and chlorine from the AMT constructs to acceptable metabolic levels while preserving the structural integrity of the AMT construct with the polymer. We expect that polymerized AMT construct implants will remain essentially unmodified in cellular environments in vitro and in vivo because of the chemical and thermal stability of the PEDOT polymer depositions, providing the basis for applications in surgical reconstructions, especially in peripheral nerve repairs and as biosynthetic prostheses. 


\section{ACKNOWLEDGMENTS}

We thank the University of Michigan Electron Microbeam Analysis Laboratory for access to its facilities. This report is presented as part of the research efforts within the Army Research Office Multidisciplinary University Research Initiative award on Bio-Integrating Structural and Neural Prosthetic Materials (W911NF-06-1-0218), and we gratefully acknowledge the funding provided.

\section{REFERENCES}

1. Aoki, T., Tanino, M. Sanui, K., Ogata, N., and Kumkura, K. Secretory function of adrenal chromaffin cells cultured on polypyrrole films. Biomaterials 17, 1971, 1996.

2. George, P.M., Lyckman, A.W., LaVan, D.A., Hegde, A., Leung, Y., and Avasare, R. Fabrication and biocompatibility of polypyrrole implants suitable for neural prosthetics. Biomaterials 26, 3511, 2005.

3. Yang, J., Kim, D.H., Hendricks, J.L., Leach, M., Northey, R., and Martin, D.C. Ordered surfactant-templated poly $(3,4-$ ethylenedioxythiophene) (PEDOT) conducting polymer on microfabricated neural probes. Acta Biomater 1, 125, 2005.

4. Xiao, Y.H., Cui, X.Y., Hancock, J.M., Bouguettaya, M.B., Reynolds, J.R., and Martin, D.C. Electrochemical polymerization of poly(hydroxymethylated-3,4-ethylenedioxythiophene) (PEDOT-MeOH) on multichannel neural probes. Sens Actuators B Chem 99, 437, 2004.

5. Heywang, G., and Jonas, F. Poly(alkylenedioxythiophene)snew, very stable conducting polymers. Adv Mater 4, 116, 1992.

6. Kirchmeyer, S. and Reuter, K. Scientific importance, properties and growing applications of poly(3,4-ethylenedioxythiophene). J Mater Chem 15, 2077, 2005.

7. Pei, Q., Zuccarello, G., Ahlskog, M., and Inganas, O. Electrochromic and highly stable poly(3,4-ethylenedioxythiophene) switches between opaque blue-black and transparent sky blue. Polymer 35, 1347, 1994.

8. Winter, I., Reese, C., Hormes, J., Heywang, G., and Jonas, F. The thermal ageing of poly(3,4-ethylenedioxythiophene). An investigation by X-ray absorption and X-ray photoelectron spectroscopy. Chem Phys 194, 207, 1995.

9. Schmidt, C.E., Shastri, V.R., Vacanti, J.P., and Langer, R. Stimulation of neurite outgrowth using an electrically conducting polymer. Proc Natl Acad Sci U S A 94, 8948, 1997.

10. Khor, E., Li, H.C., and Wee, A. In situ polymerization of pyrrole in animal tissue in the formation of hybrid biomaterials. Biomaterials 16, 657, 1995.

11. Eaglstein, W.H. and Falanga, V. Tissue engineering and the development of Apligraf a human skin equivalent. Adv Wound Care 11, 1, 1998.

12. Weber, R.A., Breidenbach, W.C., Brown, R.E., Jabaley, M.E., and Mass, D.P. A randomized prospective study conduits for digital nerve reconstruction. Reconstr Surg 106, 1036, 2000.

13. Xu, C.C., Chan, R.W., Tirunagari, N. A biodegradable, acellular xenogeneic scaffold for regeneration of the vocal fold lamina propria. Tissue Eng 13, 551, 2007.
14. Bordenave, L., Fernandez, P., Remy-Zolghadri, M., Villars, S., Daculsi, R., and Midy, D. In vitro endothelialized ePTFE prostheses: clinical update 20 years after the first realization. Clin Hemorheol Microcirc 33, 227, 2005.

15. Rovak, J.M., Bishop, D.K., Boxer, L.K., Wood, S.C., Mungara, A.K., and Cederna P.S. Peripheral nerve transplantation: the role of chemical acellularization in eliminating allograft antigenicity, J Reconstr Microsurg 3, 207, 2005.

16. Urbanchek, M.G., Wolcott, T.E., Franz, M.G., Wood, S.C., Bishop, D.K., and Kuzon, W.M. Tensile properties of mouse abdominal wall repaired with acellular myofascia closely resemble those of native abdominal wall. 52nd Annual Meeting of the Plastic Surgery Research Council. June 20-23, 2006, Stanford, California.

17. Haase, S.C., Rovak, J.M., Dennis, R.G., Kuzon, W.M., and Cederna, P.S. Recovery of muscle contractile function following nerve gap repair with chemically acellularized peripheral nerve grafts. J Reconstr Microsurg 19, 241, 2003.

18. Hong, K.H., Oh, K.W., and Kang, T.J. Preparation and properties of electrically conducting textiles by in situ polymerization of poly(3,4-ethylenedioxythiophene). J Appl Polym Sci 97, 1326, 2005.

19. Furman, N.H. Standard Methods of Chemical Analysis, $6^{\text {th }}$ Edition, Princeton, NJ: Van Nostrand, 1962.

20. Smela, E., Conjugated polymer actuators for biomedical applications. Adv Mater 15, 481, 2003.

21. Groenendaal, L., Zotti, G., Aubert, P-H., Waybright, S.M., and Reynolds, J.R. Electrochemistry of poly(3,4alkylenedioxythiophene) derivatives. Adv Mater 15, 855, 2003.

22. Corradi, R., and Armes, S.P. Chemical synthesis of poly(3,4ethylenedioxythiophene). Synth Metals 84, 453, 1997.

23. Jonas, F., Heywang, G., Schmitberg, W., Heinze, J., and Dietrich, M. US Patent 5,035,926, 1991.

24. International Agency for Research on Cancer (IARC). Monographs Program on the Evaluation of Carcinogenic Risks to Humans (2004) for methyl methanesulfonate. 71, 1059, 1999.

25. Glowienkea, S., Frieauffa, W., Allmendingerb, T., Martusa, H-J., Sutera, W., and Muellera, L. Structure-activity considerations and in vitro approaches to assess the genotoxicity of 19 methane-, benzene- and toluenesulfonic acid esters. Mutat Res 581, 23, 2005.

26. Kudoh, Y., Akami, K., and Matsuya, Y. Chemical polymerization of 3,4-ethylenedioxythiophene using an aqueous medium containing an anionic surfactant. Synth Metals 98, 65, 1998.

27. Rumbau, V., Pomposo, J.A., Eleta, A., Rodriguez, J., Grande, H., Mecerreyes, D., and Ochoteco, E. First enzymatic synthesis of water-soluble conducting poly(3,4-ethylenedioxythiophene). Biomacromolecules 8, 315, 2007.

28. Winther-Jensen, B., and West, K. Stability of highly conductive poly-3,4-ethylene-dioxythiophene. React Func Poly 66, 479, 2006.

29. Davies, F.F. The origin of pegylation. Adv Drug Deliv Rev 54, 457, 2000.

30. Dietrich, M., Heywang, G., Jonas, F., and Hemze, J. Electrochemical and spectroscopic characterization of polyalkylenedioxythiophenes J Electroanal Chem 87, 369, 1994.

31. Yamamoto, T., and Abla, M. Synthesis of non-doped poly 3,4-ethylenedioxythiophene and its spectroscopic data. Synth Metals 100, 237, 1999. 
32. Roncali, J., Garreau, R., Delabouglise, D., Garnier, F., Lemaire, M. Modification of the structure and electrochemical properties of poly(thiophene) by ether groups. J Chem Soc Chem Commun 679, 1989.

33. Monticello, D.J. Biodesulfurization and the upgrading of petroleum distillates. Curr Opin Biotechnol 11, 540, 2000.

34. Dansette, P.M., Bertho, G., and Mansuy, D. First evidence that cytochrome P450 may catalyze both S-oxidation and epoxidation of thiophene derivatives. Biochem Biophys Res Commun 338, 450, 2005.

35. Yamamoto, T., Morita, A., Miyazaki, Y., Maruyama, T., Wakayama, H., Zhou, Z.H., Nakamura, Y., Kanbara, T., Sasaki, S., and Kubota, K. Preparation of $\pi$-conjugated poly(thiophene-2,5-diyl), poly ( $p$-phenylene) and related polymers using zerovalent nickel complexes. Linear structure and properties of the $\pi$-conjugated polymers. Macromolecules 25, 1214, 1992.

36. Andrews, N.C. Disorders of iron metabolism, N Engl J Med 342, 364, 2000.

Address reprint requests to: David Martin, Ph.D. Department of Materials Science and Engineering

University of Michigan 2644 CSE Building Ann Arbor, MI 48109

E-mail: milty@umich.edu 